$10.31341 /$ jios.45.2.5

UDC 004.81:159.953.5

OOpen Access

Original Scientific Paper

\title{
Spectral Indexes Evaluation for Satellite Images Classification using CNN
}

\author{
Vladyslav Yaloveha \\ Faculty of Computer and Information Technologies \\ National Technical University "KhPI”, Kharkiv, Ukraine
}

vladyslavyaloveha@gmail.com

Daria Hlavcheva

Faculty of Computer and Information Technologies

National Technical University "KhPI”, Kharkiv, Ukraine

dariahlavcheva@gmail.com

Andrii Podorozhniak

andriipodorozhniak@gmail.com

Faculty of Computer and Information Technologies

National Technical University "KhPI”, Kharkiv, Ukraine

\begin{abstract}
Deep learning approaches are applied for a wide variety of problems, they are being used in the remote sensing field of study and showed high performance. Recent studies have demonstrated the efficiency of using spectral indexes in classification problems, because of accuracy and F1 score increasing in comparison with the usage of only RGB channels. The paper studies the problem of classification satellite images on the EuroSAT dataset using the proposed convolutional neural network. In the research set of the most used spectral indexes have been selected and calculated on the EuroSAT dataset. Then, a novel comparative analysis of spectral indexes was carried out. It has been established that the most significant set of indexes (NDVI, NDWI, GNDVI) increased classification accuracy from $64.72 \%$ to $84.19 \%$ and F1 score from $63.89 \%$ to $84.05 \%$. The biggest improvement was obtained for River, Highway and PermanentCrop classes.
\end{abstract}

Keywords: Earth remote sensing, deep learning, spectral indexes, convolutional neural networks, EuroSAT

\section{Introduction}

Remote sensing is a technique that is used for monitoring the physical characteristics of objects and capturing information remotely using sensors. Remote sensing is based on measuring radiation at a distance.

In recent years, the availability of remote images regularly acquired by satellites has been increased due to high-resolution and multi-spectral imaging Landsat and ESA Sentinel missions. These missions opened opportunities for collecting images in datasets and using them for solving issues. 
Deep Learning (DL) approaches including neural networks are applied for a wide variety of problems, popular in the remote sensing field of study and showed high performance. These models learn features from the input data automatically, unlike classical machine learning algorithms that usually require data preprocessing.

Deep learning was successfully used for land [1] and fire hazardous forest areas classification [2], [3], object detection [4], deforestation problems [5], observation of global changes, agriculture analysis [6], and healthcare issues [7]. Among all the DL models, convolutional neural networks are becoming increasingly ubiquitous because of their remarkable results in many domains, including remote sensing issues. This is because of the image's stationary property which states that contents retrieved from one part of an image can also be applied to another part of the image [8].

The novelty of the research is investigating the significant set of spectral indexes for multiclass classification of satellite images on the EuroSAT dataset and proposed convolutional neural network, that could increase classification accuracy and F1 score in comparison with classical true color (RGB) images.

\section{Remote Sensing Images}

Landsat8 and Sentinel2 are multispectral instruments that are publicly available and provide remote sensing images of Earth's land surfaces continuously. Landsat 8 that was launched in 2013 according to the Landsat Earth observation program (since 1972 [9]) in NASA and USGS collaboration, acquires global moderate-resolution measurements of the Earth's terrestrial and polar regions in the visible, near-infrared, short wave, and thermal infrared [10].

The Sentinel-2 mission provides for a combination of two satellites - Sentinel-2A and Sentinel-2B - equipped with identical Multispectral Instruments (MSI). Sentinel2A was launched in June 2015. The MSI has a very wide field of view (290 km swath width, which is significantly larger than the $185 \mathrm{~km}$ of the OLI) [11]. Despite Landsat 8 supplies images in 11 different bands with $(30-15 \mathrm{~m})$ spatial resolution and global coverage approximately every three days, the Sentinel-2 Multi-Spectral Instrument features 13 spectral bands spanning from the visible and near-infrared (VNIR) to the short-wave infrared (SWIR), featuring 4 spectral bands at $10 \mathrm{~m}, 6$ bands at $20 \mathrm{~m}$ and 3 bands at $60 \mathrm{~m}$ spatial resolution and 10 days minimum revisit time at the equator Aerosols, Cirrus and Water vapor bands could be used for the correction of atmospheric effects [12], [13]. Higher image resolution allows to figure out more precise calculations.

Getting periodically remote sensing images permitted forming them in datasets for next usage in neural networks. Some of the open-sourced remote sensing datasets are presented in Table 1.

\begin{tabular}{|l|c|c|c|c|}
\hline & Dataset Name & Type & Number of images & Reference \\
\hline $\mathbf{1}$ & UC Merced & RGB & 2,100 & {$[14]$} \\
\hline $\mathbf{2}$ & RSSCN7 & RGB & 2,800 & {$[15]$} \\
\hline $\mathbf{3}$ & AID & RGB & 10,000 & {$[16]$} \\
\hline $\mathbf{4}$ & RSI-CB & RGB & 36,707 & {$[17]$} \\
\hline
\end{tabular}




\begin{tabular}{|l|c|c|c|c|}
\hline $\mathbf{5}$ & PatternNet & RGB & 30,400 & {$[18]$} \\
\hline $\mathbf{6}$ & NWPU-RESISC45 & RGB & 31,500 & {$[19]$} \\
\hline $\mathbf{7}$ & EuroSAT & Multispectral & 27,000 & {$[20]$} \\
\hline
\end{tabular}

Table 1. Comparison remote sensing datasets.

UC Merced - classical remote sensing dataset that consists of 21 classes with 256x256 pixels image size. All images are presented in true color (RGB). The dataset is small (only 100 images per class). PatternNet and NWPU-RESISC45 datasets include a few numbers of images per class but with high-resolution. RSI-CB contains 45 categories and more than 36,000 images with up to $3 \mathrm{~m}$ spatial resolution. The AID remote sensing image dataset is based on Google Earth images. It contains 10,000 images with 30 classes and up to 400 images per class.

Among considered remote sensing datasets EuroSAT is the most recent (published in 2019) and has all possible multispectral bands. It consists of 27,000 images across 34 European countries: Austria, Belarus, Belgium, Bulgaria, Cyprus, Czech Republic, Denmark, Estonia, Finland, France, Germany, Greece, Hungary, Iceland, Ireland, Italy, Latvia, Lithuania, Luxembourg, Macedonia, Malta, Republic of Moldova, Netherlands, Norway, Poland, Portugal, Romania, Slovakia, Slovenia, Spain, Sweden, Switzerland, Ukraine, and United Kingdom. The dataset is separated into 10 different classes with 2,000 to 3,000 images per class (see Fig. 1). Images size is $64 \times 64$ pixels, spatial resolution is up to 10 meters per pixel [20]. The dataset is slightly imbalanced for Highway, Industrial, Pasture, PermanentCrop, and River classes.

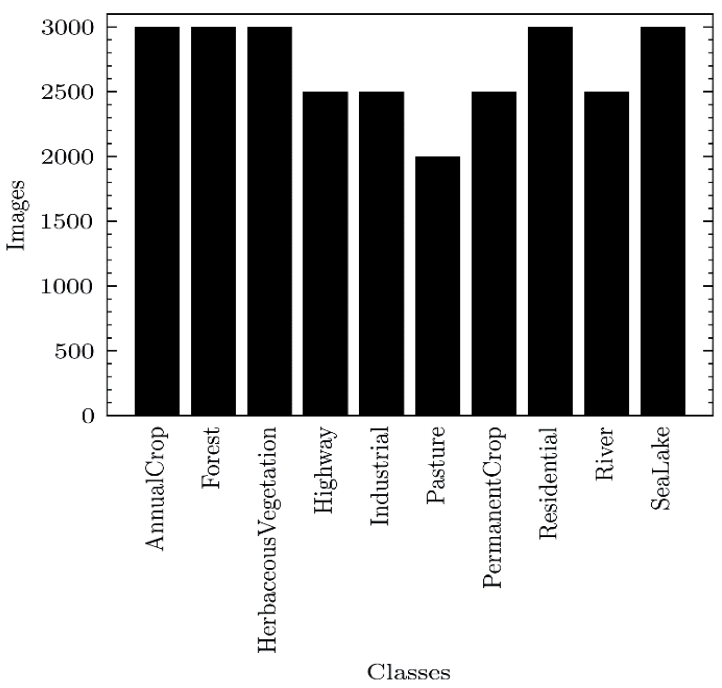

Figure 1. EuroSAT dataset distribution by classes. 


\section{Spectral indexes}

Deep learning allows computational models, that are composed of multiple processing layers to learn representations of data with multiple levels of abstraction [21].

However, sometimes these algorithms are not able to find all significant representations from given RGB-like input images and their efficiency of the DL could be improved due to adding more information into the system. To analyze specific land surface changes, land use/land cover change, or urban expansion, a common approach involves the processing and transformation of the raw spectral information contained in remotely sensed images [22]. For this purpose, we can calculate spectral indexes (SI) from available spectral bands to generate additional features for the deep learning neural network.

The vegetation spectral indexes are features that represent directly or indirectly the biochemical and biophysics vegetation or crop characteristics. These indexes are obtained through information provided by spectral sensors [23]. Some scientists around the world have demonstrated the efficiency and potential of generating vegetation indexes for the estimation of useful variables for agricultural activities. Spectral indexes are used for improving the accuracy of classification. In [24] they were applied to the problem of land cover classification of tree species and forest change detection.

Vegetation indexes help to estimate changes in the environment caused by growth in the human population and decreasing natural resources [25]. Some studies have focused on the production of thematic maps using remote sensing data and they reported that remote sensing provides effective support to the management of agricultural fields by providing spatial and temporal information for vegetation monitoring over large areas [26]. This research effectively applied spectral indexes for monitoring agriculture fields and generated a classification map. Spectral indexes are successfully used for analyzing the urbanization problem and got high performance [27].

Among a wide range of spectral indexes proposed in the papers mentioned above, we selected 10 and calculated each of them for all images in the EuroSAT dataset (Table 2).

\begin{tabular}{|c|c|c|c|c|}
\hline & Spectral Index & Abbreviation & Equation & $\begin{array}{c}\text { Refer } \\
\text { ence }\end{array}$ \\
\hline $\mathbf{1}$ & $\begin{array}{c}\text { Normalized } \\
\text { Difference } \\
\text { Vegetation } \\
\text { Index }\end{array}$ & NDVI & $\frac{\rho_{\text {nir }}-\rho_{\text {red }}}{\rho_{\text {nir }}+\rho_{\text {red }}}$ & {$[28]$} \\
\hline $\mathbf{2}$ & $\begin{array}{c}\text { Normalized } \\
\text { Difference } \\
\text { Water Index }\end{array}$ & NDWI & $\frac{\rho_{\text {nir }}-\rho_{\text {swir }}}{\rho_{\text {nir }}+\rho_{\text {swir }}}$ & {$[29]$} \\
\hline
\end{tabular}

${ }^{1}$ Where $\rho_{i}$-reflectance value of $i$-th band. 


\begin{tabular}{|c|c|c|c|c|}
\hline 3 & $\begin{array}{l}\text { Plant } \\
\text { Senescence } \\
\text { Reflectance } \\
\text { Index }\end{array}$ & PSRI & $\frac{\rho_{\text {red }}-\rho_{\text {green }}}{\rho_{\text {nir }}}$ & [30] \\
\hline 4 & $\begin{array}{l}\text { Normalized } \\
\text { Difference } \\
\text { Green NDVI } \\
\end{array}$ & GNDVI & $\frac{\rho_{\text {nir }}-\rho_{\text {green }}}{\rho_{\text {nir }}+\rho_{\text {green }}}$ & [31] \\
\hline 5 & $\begin{array}{l}\text { Global } \\
\text { Environmental } \\
\text { Monitoring } \\
\text { Index }\end{array}$ & GEMI & $\begin{array}{c}\eta(1-0.25 \eta)-\frac{\rho_{\text {red }}-0.125}{1-\rho_{\text {red }}}, \\
\text { where } \eta=\frac{2\left(\rho_{\text {nir }}^{2}-\rho_{\text {red }}^{2}\right)+1.5 \rho_{\text {nir }}+0.5 \rho_{\text {red }}}{\rho_{\text {nir }}+\rho_{\text {red }}+0.5}\end{array}$ & [32] \\
\hline 6 & $\begin{array}{l}\text { Normalized } \\
\text { Difference } \\
\text { Pond Index }\end{array}$ & NDPI & $\frac{\rho_{\text {swir }}-\rho_{\text {green }}}{\rho_{\text {swir }}+\rho_{\text {green }}}$ & [33] \\
\hline 7 & $\begin{array}{l}\text { Water Band } \\
\text { Index }\end{array}$ & WBI & $\frac{\rho_{\text {blue }}-\rho_{\text {red }}}{\rho_{\text {blue }}+\rho_{\text {red }}}$ & [34] \\
\hline 8 & $\begin{array}{l}\text { Enhanced } \\
\text { Vegetation } \\
\text { Index } \\
\end{array}$ & EVI & $2.5 \cdot \frac{\rho_{\text {nir }}-\rho_{\text {red }}}{\rho_{\text {nir }}+6 \rho_{\text {red }}-7.5 \rho_{\text {blue }}+1}$ & {$[35]$} \\
\hline 9 & $\begin{array}{c}\text { Modified } \\
\text { Simple Ratio }\end{array}$ & MSR & $\left(\frac{\rho_{\text {nir }}}{\rho_{\text {red }}}-1\right): \sqrt{\frac{\rho_{\text {nir }}}{\rho_{\text {red }}}+1}$ & {$[36]$} \\
\hline 10 & Simple Ratio & SR & $\frac{\rho_{\text {nir }}}{\rho_{\text {red }}}$ & [37] \\
\hline
\end{tabular}

Table 2. Spectral indexes.

\section{Convolutional neural networks (CNNs)}

In recent time, deep learning has brought revolutionary achievements in many applications, including the classification of remote sensing images. The state-of-theart results have been achieved by the Convolutional Neural Networks (CNNs) due to their structure being able to extract more hidden and deeper features than classic machine learning methods [38]. This concept lies at the basis of many deep learning algorithms: models (networks) composed of many layers that transform input data (e.g., images) to outputs (e.g., disease present/absent) while learning increasingly high-level features [39].

CNN architecture consists of layers, namely, convolutional layers, max-pooling, and fully connected layers. The main idea of a convolutional layer is extracting significant features from previous layers of the network and generating feature maps as an output. There is a kernel that is defined as a matrix and saves information of an object. Feature maps values bind with the kernel. Features encode useful information about a considered object from simple to most generic (for instance, image color, 
orientation). The sub-sampling layer also called as pooling layer, is mainly implemented to reduce the size of the image data and the variance of features extracted from the convolutional layer with the retention of the geometry of the input data [8].

For the current research, we propose convolutional neural network architecture presented in Fig. 2. It consists of sequences of Convolutional and MaxPooling layers. At the final stage of the neural network, there are two Dense layers with a softmax activation function that represents classification probabilities for each class in the EuroSAT dataset. For preventing overfitting problem BatchNormalization (after every combination of convolutional and pooling layers) and Dropout (after fully connected layer with rate 0.2 ) layers were used.

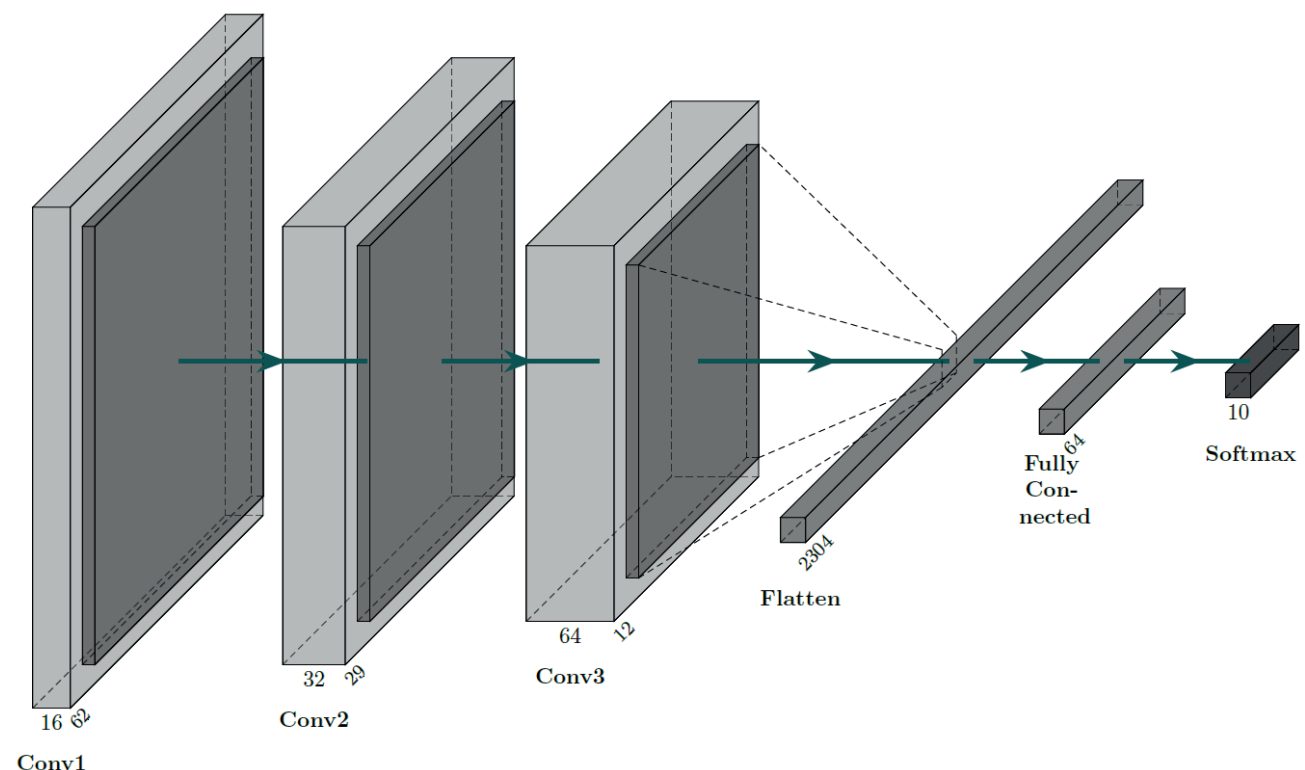

Figure 2. The architecture of the proposed convolutional neural network.

For experiments, we had chosen $\operatorname{Re} \operatorname{Lu}(x)=\max (0, x)$ activation function among neural network layers and softmax $s(\vec{x})_{i}=\frac{e^{x_{i}}}{\sum_{j=1}^{10} e^{x_{j}}}$, where $\vec{x}=\left(x_{0} \ldots x_{10}\right)$ in the end. RMSprop learning algorithm with $1 e-6$ learning rate was selected. The learning algorithm is employed to decrease the categorical cross-entropy loss (which relates to a probability over the classes for each image and is suitable for multiclass classification issues) [40]. While training we used EarlyStopping [41] technique with the patience of 5 that monitored accuracy on the validation subset. That is why the number of epochs varies for every experiment.

To evaluate the considered models the classification accuracy metric and F1 score were selected. Accuracy is one of the most commonly used measures for classification 
performance, and it is defined as a ratio between the correctly classified samples to the total number of samples:

$$
\text { Accuracy }=\frac{T P+T N}{T P+T N+F P+F N},
$$

where correctly classified positive sample is called (TP), classified as negative false negative (FN). If the sample is negative and it is classified as negative it is considered as true negative (TN); if it is classified as positive, it is counted as false positive (FP). However, classification accuracy in multiclass tasks is sensitive to the imbalanced data [42]. It can give high results when the number of samples in one class more than in others, but it doesn't mean that a neural network could classify all classes correctly with the same value of success. F1-score represents the harmonic mean of precision and recall. The value of F-measure is ranged from zero to one, and high values indicate better classification performance. The metric is sensitive to changes in data distributions and could be used for imbalanced datasets evaluation:

$$
F_{1}=\frac{2 T P}{2 T P+F P+F N} .
$$

\section{Results and discussion}

The programming environment, for the research was the Jupyter Lab with Python 3.7. The learning frameworks Tensorflow 2.0 and Keras 2.3, and the library for working with multi-dimensional arrays Numpy 1.18 were used. Training, validation, and testing of CNN were performed on ASUS laptop. The laptop has 16Gb of RAM, one Nvidia GeForce 940MX GPU and an Intel Core i7 extreme 7th generation CPU (up to $3.5 \mathrm{GHz}$ ).

We have separated the EuroSAT dataset to train, validation, and test subsets. The train dataset contains $70 \%$ of all images, validation and test datasets consist of $15 \%$ each. Examples of images by classes are shown in Fig. 3.
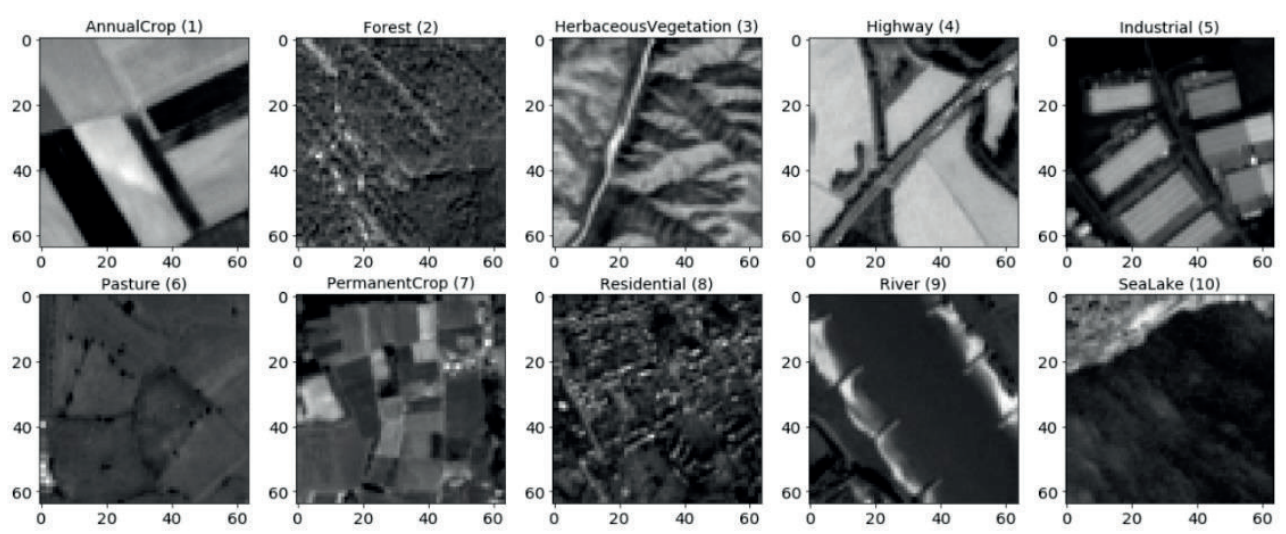

Figure 3. Image examples of the EuroSAT dataset. 
We have carried out a set of experiments using the grid search to find the best suitable combination of spectral indexes that allowed to improve classification accuracy and F1 score on a given dataset.

The graphs in Fig. 4-5 show the accuracy classification dependence on epochs for RGB images and their combinations with indexes. The highest growth was for RGB NDVI, NDWI, and GNDVI combination on train and validation datasets.

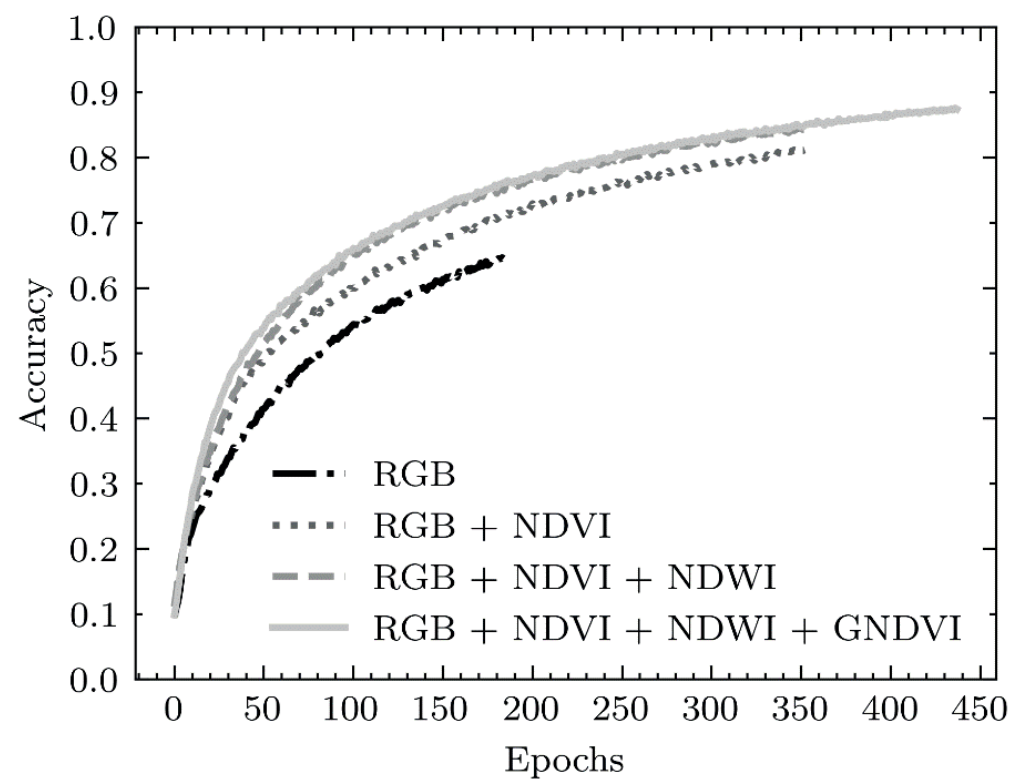

Figure 4. Classification accuracy on train data.

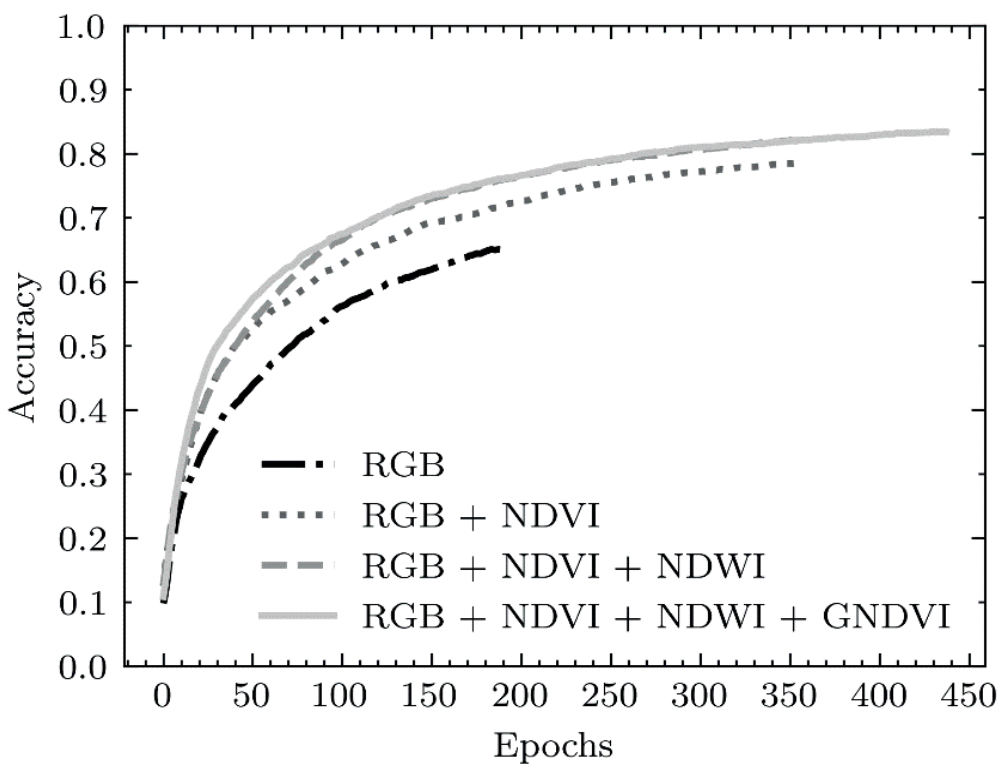

Figure 5. Classification accuracy on validation data. 


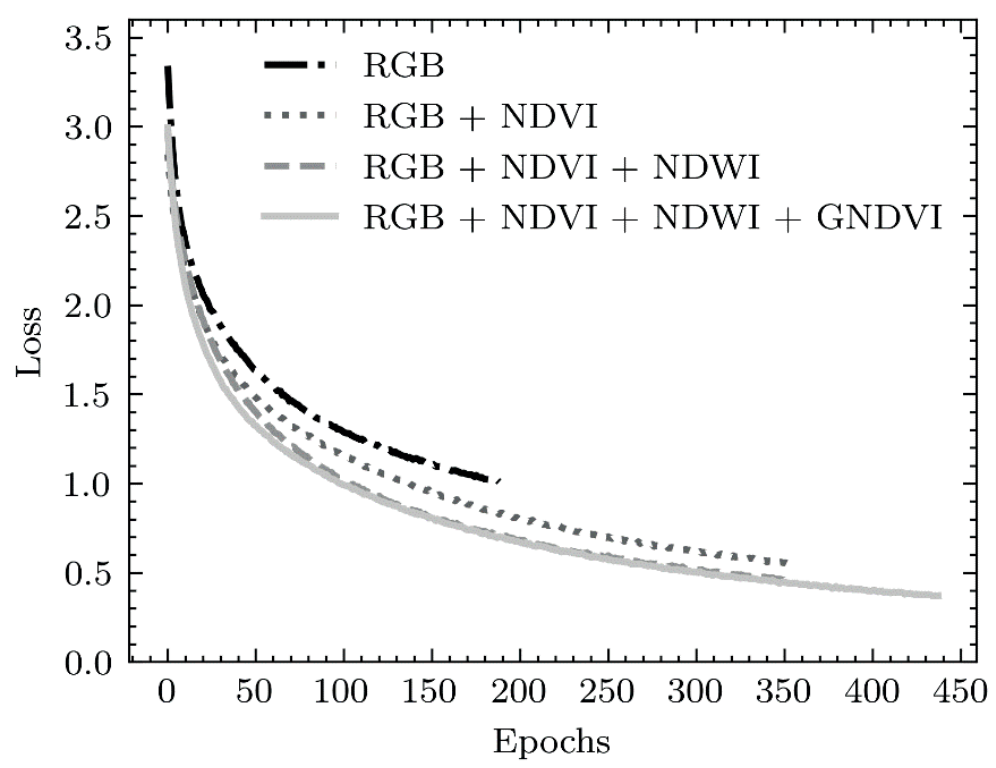

Figure 6. Classification loss on train data.

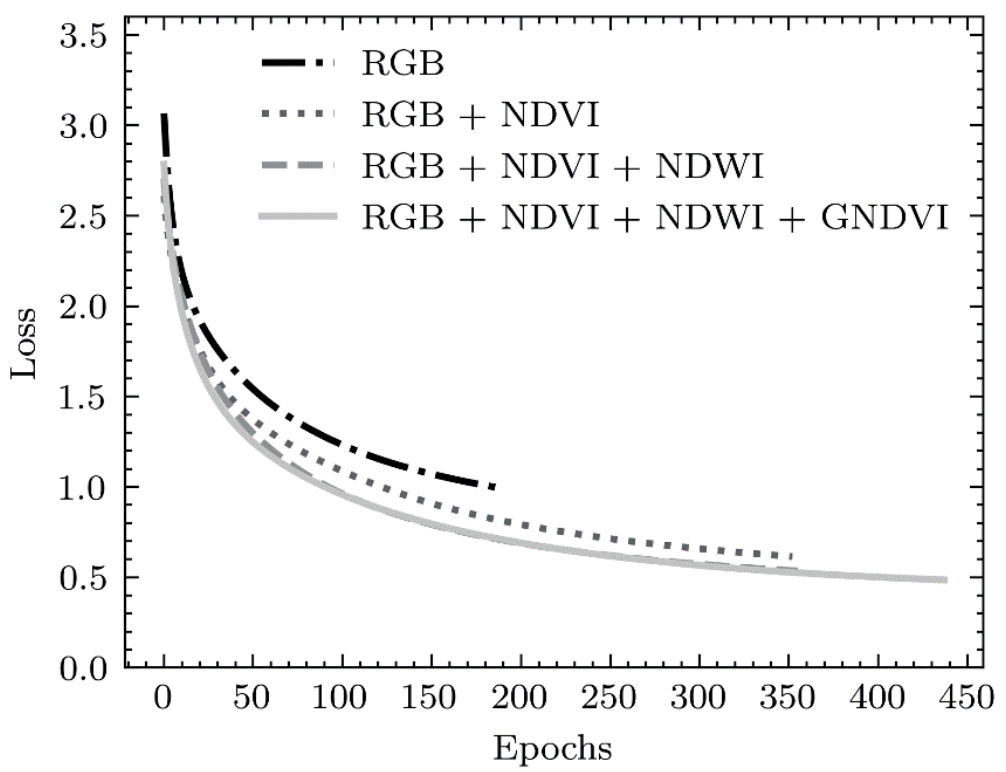

Figure 7. Classification loss on validation data.

The graphs in Fig. 6-7 present loss dependence on epochs. A consistent drop of loss on train and validation datasets was observed. Even on the first fifty epochs adding indexes helped to decrease loss function compared to images with RGB channels. The combination of NDVI, NDWI, and GNDVI indexes showed a more sharped drop on early epochs. 
Table 3 outlines the process of finding the best set of spectral indexes. As a baseline in the first experiment, only RGB images were selected. The classification accuracy and F1 scores were $64.72 \%$ and $63.89 \%$ respectively. In the next steps, we added indexes one by one and monitored selected metrics. In the last Step 4, the improvement was not achieved, so the grid search had been stopped. The maximum obtained accuracy is $84.19 \%$, and the F1 score is $84.05 \%$.

According to the grid search results, it had been established that for the EuroSAT dataset most relevant indexes combination is NDVI, NDWI and GNDVI together with RGB channels.

\begin{tabular}{|c|c|c|c|c|c|c|c|c|c|c|c|}
\hline & Step & \multicolumn{2}{|c|}{$\mathbf{0}$} & \multicolumn{2}{|c|}{$\mathbf{1}$} & \multicolumn{2}{|c|}{$\mathbf{2}$} & \multicolumn{2}{|c|}{$\mathbf{3}$} & \multicolumn{2}{|c|}{4} \\
\hline & $\begin{array}{c}\text { Combin } \\
\text { ation }\end{array}$ & Acc $^{2}$ & F1 & Acc & F1 & Acc & F1 & Acc & F1 & Acc & F1 \\
\hline $\mathbf{1}$ & RGB & $\mathbf{6 4 . 7 2}$ & $\mathbf{6 3 . 8 9}$ & - & - & - & - & - & - & - & - \\
\hline $\mathbf{2}$ & NDVI & - & - & $\mathbf{7 9 . 0 9}$ & $\mathbf{7 8 . 8 8}$ & - & - & - & - & - & - \\
\hline $\mathbf{3}$ & NDWI & - & - & 74.59 & 74.21 & $\mathbf{8 1 . 4 8}$ & $\mathbf{8 1 . 3 0}$ & - & - & - & - \\
\hline $\mathbf{4}$ & GNDVI & - & - & 77.00 & 76.69 & 81.07 & 80.92 & $\mathbf{8 4 . 1 9}$ & $\mathbf{8 4 . 0 5}$ & - & - \\
\hline $\mathbf{5}$ & PSRI & - & - & 69.87 & 69.53 & 81.31 & 81.12 & 80.61 & 80.43 & 78.59 & 78.30 \\
\hline $\mathbf{6}$ & GEMI & - & - & 72.13 & 71.68 & 77.61 & 77.36 & 80.56 & 80.28 & 78.19 & 77.87 \\
\hline $\mathbf{7}$ & NDPI & - & - & 73.98 & 73.47 & 80.78 & 80.52 & 82.54 & 82.33 & $\mathbf{8 3 . 6 3}$ & $\mathbf{8 3 . 4 4}$ \\
\hline $\mathbf{8}$ & WBI & - & - & 72.30 & 71.65 & 80.80 & 80.69 & 82.04 & 81.85 & 82.43 & 82.26 \\
\hline $\mathbf{9}$ & EVI & - & - & 68.22 & 67.51 & 79.65 & 79.43 & 79.15 & 78.88 & 77.50 & 77.08 \\
\hline $\mathbf{1 0}$ & MSR & - & - & 76.93 & 76.65 & 76.81 & 76.51 & 81.50 & 81.35 & 81.46 & 81.28 \\
\hline $\mathbf{1 1}$ & SR & - & - & 78.54 & 78.28 & 79.74 & 79.54 & 78.76 & 78.50 & 82.06 & 81.87 \\
\hline
\end{tabular}

Table 3. Experimental results on EuroSAT dataset, \%.

Although on Step 3 we achieved F1 score of $84.05 \%$ it is useful to analyze metrics in the context of every class in the dataset (Table 4). Despite, on zero step F1 score is $63.89 \%$ for River, Highway, PermanentCrop and HerbaceousVegetation classes the metric even low than $50 \%$ which means that the neural network couldn't retrieve significant features from input information and correctly classify images belonging to the classes.

\begin{tabular}{|l|c|c|c|c|c|c|}
\hline & Class & RGB & $\begin{array}{c}\text { RGB + } \\
\text { NDVI }\end{array}$ & $\begin{array}{c}\text { RGB + } \\
\text { NDVI + } \\
\text { NDWI }\end{array}$ & $\begin{array}{c}\text { RGB + NDVI + } \\
\text { NDWI + GNDVI }\end{array}$ & $\Delta^{3}$ \\
\hline $\mathbf{1}$ & River & 43.46 & 77.47 & 85.63 & 88.78 & 45.32 \\
\hline $\mathbf{2}$ & Highway & 43.26 & 62.99 & 66.59 & 73.17 & 29.91 \\
\hline $\mathbf{3}$ & PermanentCrop & 37.97 & 60.67 & 62.85 & 67.52 & 29.55 \\
\hline $\mathbf{4}$ & Industrial & 64.46 & 85.74 & 87.23 & 88.95 & 24.49 \\
\hline $\mathbf{5}$ & HerbaceousVegetation & 49.42 & 67.91 & 66.96 & 71.34 & 21.92 \\
\hline $\mathbf{6}$ & AnnualCrop & 66.42 & 75.93 & 80.22 & 84.72 & 18.30 \\
\hline
\end{tabular}

\footnotetext{
${ }^{2}$ Acc - classification accuracy.

$3 \Delta$-Difference between first and last columns.
} 


\begin{tabular}{|c|c|c|c|c|c|c|}
\hline $\mathbf{7}$ & Pasture & 64.01 & 74.87 & 74.30 & 77.02 & 13.01 \\
\hline $\mathbf{8}$ & Forest & 82.92 & 89.98 & 93.11 & 93.55 & 10.63 \\
\hline $\mathbf{9}$ & Residential & 84.06 & 92.46 & 93.84 & 93.46 & 9.40 \\
\hline $\mathbf{1 0}$ & SeaLake & 91.86 & 94.68 & 96.07 & 96.67 & 4.81 \\
\hline
\end{tabular}

Table 4. F1 score on EuroSAT dataset by classes, \%.

Adding indexes to RGB channels allowed to increase the F1 score. $\Delta$ shows improvement between baseline experiment and after carrying out a grid search. As we can see from Table 4 F1 score increased for all classes in the EuroSAT dataset. The biggest improvement was for River (45.32\%), Highway (29.91\%), and PermanentCrop (29.55\%) classes. At the same time, SeaLake showed only $4.81 \%$ up. For the Residential class adding the GNDVI index slightly decreased the F1 score $(0.38 \%)$.

In [24] was also found that the use of spectral indexes improved the classification performance applied to the problem of classification tree species. In [26] authors got improvements in classification for agriculture problems. They got an overall accuracy of $93.1 \%$ based on the reflectance at 4 bands and 8 spectral indexes. On the other hand, for research were used 5 different classes. We expect increasing the F1 score and classification accuracy after optimizing hyperparameters and the architecture of the convolutional neural network.

\section{Conclusions}

Analyzing and classifying remote sensing images applied in a wide range of issues such as land use, deforestation, climate, urbanization, and agriculture changes. Many research papers demonstrate the efficiency of using spectral indexes. In particular, deep learning neural networks can extract significant features from input data, provided in the form of spectral images.

EuroSAT dataset provides 27,000 remote sensing images and contains a full range of Sentinel2 spectral bands. Such variety allows calculating spectral indexes.

In this paper, we have addressed the challenge of multiclass classification EuroSAT remote sensing. The experiments based on grid search using a deep learning approach were conducted. It is obtained that adding indexes to an input of the proposed convolutional neural network increase classification accuracy and F1 score in comparison with the baseline experiment. The best combination of indexes is NDVI, NDWI, GNDVI with RGB channels.

Our findings also indicate that spectral indexes showed high performance on River (45.32\%), Highway (29.91\%) and PermanentCrop (29.55\%) classes.

The overall accuracy of classification increased from $64.72 \%$ to $84.19 \%$. As a result of the experiments, the indexes combination, with an F1 score of $84.05 \%$, outperformed single RGB images in the baseline experiment. 


\section{References}

[1] N. Kussul, M. Lavreniuk, S. Skakun, and A. Shelestov, "Deep learning classification of land cover and crop types using remote sensing data," IEEE Geoscience and Remote Sensing Letters, vol. 14, no. 5, pp. 778-782, 2017.

[2] H. Kuchuk, A. Podorozhniak, D. Hlavcheva, and V. Yaloveha, "Application of Deep Learning in the Processing of the Aerospace System's Multispectral Images," in Handbook of Research on Artificial Intelligence Applications in the Aviation and Aerospace Industries: IGI Global, 2020, pp. 134-147.

[3] V. Yaloveha, D. Hlavcheva, A. Podorozhniak, and H. Kuchuk, "Fire hazard research of forest areas based on the use of convolutional and capsule neural networks," in 2019 IEEE 2nd Ukraine Conference on Electrical and Computer Engineering (UKRCON), 2019: IEEE, pp. 828832.

[4] Y. Zhong, X. Han, and L. Zhang, "Multi-class geospatial object detection based on a position-sensitive balancing framework for high spatial resolution remote sensing imagery," ISPRS journal of photogrammetry and remote sensing, vol. 138, pp. 281-294, 2018.

[5] K. Isaienkov, M. Yushchuk, V. Khramtsov, and O. Seliverstov, "Deep Learning for Regular Change Detection in Ukrainian Forest Ecosystem With Sentinel-2," IEEE Journal of Selected Topics in Applied Earth Observations and Remote Sensing, 2020.

[6] V. Mazzia, A. Khaliq, and M. Chiaberge, "Improvement in Land Cover and Crop Classification based on Temporal Features Learning from Sentinel-2 Data Using Recurrent-Convolutional Neural Network (RCNN)," Applied Sciences, vol. 10, no. 1, p. 238, 2020.

[7] D. Hlavcheva, V. Yaloveha, A. Podorozhniak, and H. Kuchuk, "Tumor Nuclei Detection in Histopathology Images Using R-CNN", in CEUR Workshop Proceedings, 2020: IEEE, vol. 1, pp. 63-74.

[8] S. S. Virnodkar, V. K. Pachghare, V. Patil, and S. K. Jha, "CaneSat dataset to leverage convolutional neural networks for sugarcane classification from Sentinel-2, "Journal of King Saud University-Computer and Information Sciences, 2020.

[9] H. J. Kramer, Observation of the Earth and its Environment: Survey of Missions and Sensors. Springer Science \& Business Media, 2002.

[10] D. P. Roy et al., "Landsat-8: Science and product vision for terrestrial global change research, "Remote sensing of Environment, vol. 145, pp. 154-172, 2014. 
[11] E. Mandanici and G. Bitelli, "Preliminary comparison of sentinel-2 and landsat 8 imagery for a combined use, "Remote Sensing, vol. 8, no. 12, p. 1014, 2016.

[12] H. Van der Werff and F. Van der Meer, "Sentinel-2A MSI and Landsat 8 OLI provide data continuity for geological remote sensing, "Remote sensing, vol. 8, no. 11, p. 883, 2016.

[13] M. Drusch et al., "Sentinel-2: ESA's optical high-resolution mission for GMES operational services, "Remote sensing of Environment, vol. 120, pp. 25-36, 2012.

[14] Y. Yang and S. Newsam, "Bag-of-visual-words and spatial extensions for land-use classification," in Proceedings of the 18th SIGSPATIAL international conference on advances in geographic information systems, pp. 270-279, 2010.

[15] Q. Zou, L. Ni, T. Zhang, and Q. Wang, "Deep learning based feature selection for remote sensing scene classification, "IEEE Geoscience and Remote Sensing Letters, vol. 12, no. 11, pp. 2321-2325, 2015.

[16] G.-S. Xia et al., "AID: A benchmark data set for performance evaluation of aerial scene classification, "IEEE Transactions on Geoscience and Remote Sensing, vol. 55, no. 7, pp. 3965-3981, 2017.

[17] H. Li et al., "RSI-CB: A large scale remote sensing image classification benchmark via crowdsource data," arXiv preprint arXiv:1705.10450, 2017.

[18] W. Zhou, S. Newsam, C. Li, and Z. Shao, "PatternNet: A benchmark dataset for performance evaluation of remote sensing image retrieval, "ISPRS journal of photogrammetry and remote sensing, vol. 145, pp. 197209, 2018.

[19] G. Cheng, J. Han, and X. Lu, "Remote sensing image scene classification: Benchmark and state of the art, "Proceedings of the IEEE, vol. 105, no. 10, pp. 1865-1883, 2017.

[20] P. Helber, B. Bischke, A. Dengel, and D. Borth, "Eurosat: A novel dataset and deep learning benchmark for land use and land cover classification, "IEEE Journal of Selected Topics in Applied Earth Observations and Remote Sensing, vol. 12, no. 7, pp. 2217-2226, 2019.

[21] Y. LeCun, Y. Bengio, and G. Hinton, "Deep learning, "nature, vol. 521, no. 7553, pp. 436-444, 2015.

[22] D. Fornacca, G. Ren, and W. Xiao, "Evaluating the best spectral indices for the detection of burn scars at several post-fire dates in a mountainous region of Northwest Yunnan, China, "Remote Sensing, vol. 10, no. 8, p. 1196, 2018. 
[23] P. S. Thenkabail and J. G. Lyon, Hyperspectral remote sensing of vegetation. CRC press, 2016.

[24] M. Immitzer, M. Neuwirth, S. Böck, H. Brenner, F. Vuolo, and C. Atzberger, "Optimal Input Features for Tree Species Classification in Central Europe Based on Multi-Temporal Sentinel-2 Data, "Remote Sensing, vol. 11, no. 22, p. 2599, 2019.

[25] S. Alademomi, C. J. Okolie, O. E. Daramola, R. O. Agboola, and T. J. Salami, "Assessing the Relationship of LST, NDVI and EVI with Land Cover Changes in the Lagos Lagoon Environment, "Quaestiones Geographicae, vol. 39, no. 3, pp. 87-109, 2020.

[26] N. Kobayashi, H. Tani, X. Wang, and R. Sonobe, "Crop classification using spectral indices derived from Sentinel-2A imagery, "Journal of Information and Telecommunication, vol. 4, no. 1, pp. 67-90, 2020.

[27] S. Candiago, F. Remondino, M. De Giglio, M. Dubbini, and M. Gattelli, "Evaluating multispectral images and vegetation indices for precision farming applications from UAV images, "Remote sensing, vol. 7, no. 4, pp. 4026-4047, 2015.

[28] J. Rouse Jr, R. Haas, D. Deering, J. Schell, and J. Harlan, "Monitoring the Vernal Advancement and Retrogradation (Green Wave Effect) of Natural Vegetation.[Great Plains Corridor]," 1974.

[29] S. K. McFeeters, "The use of the Normalized Difference Water Index (NDWI) in the delineation of open water features, "International journal of remote sensing, vol. 17, no. 7, pp. 1425-1432, 1996.

[30] M. N. Merzlyak, A. A. Gitelson, O. B. Chivkunova, and V. Y. Rakitin, "Non-destructive optical detection of pigment changes during leaf senescence and fruit ripening, "Physiologia plantarum, vol. 106, no. 1, pp. 135-141, 1999.

[31] A. Gitelson and M. N. Merzlyak, "Signature analysis of leaf reflectance spectra: algorithm development for remote sensing of chlorophyll, "Journal of plant physiology, vol. 148, no. 3-4, pp. 494-500, 1996.

[32] Pinty and M. Verstraete, "GEMI: a non-linear index to monitor global vegetation from satellites, "Vegetatio, vol. 101, no. 1, pp. 15-20, 1992.

[33] J. Lacaux, Y. Tourre, C. Vignolles, J. Ndione, and M. Lafaye, "Classification of ponds from high-spatial resolution remote sensing: Application to Rift Valley Fever epidemics in Senegal, "Remote Sensing of Environment, vol. 106, no. 1, pp. 66-74, 2007.

[34] J. Peñuelas, I. Filella, C. Biel, L. Serrano, and R. Save, "The reflectance at the 950-970 $\mathrm{nm}$ region as an indicator of plant water status, "International journal of remote sensing, vol. 14, no. 10, pp. 1887-1905, 1993. 
[35] Huete, K. Didan, T. Miura, E. P. Rodriguez, X. Gao, and L. G. Ferreira, "Overview of the radiometric and biophysical performance of the MODIS vegetation indices, "Remote sensing of environment, vol. 83, no. 1-2, pp. 195-213, 2002.

[36] J. M. Chen, "Evaluation of vegetation indices and a modified simple ratio for boreal applications, "Canadian Journal of Remote Sensing, vol. 22, no. 3, pp. 229-242, 1996.

[37] G. S. Birth and G. R. McVey, "Measuring the color of growing turf with a reflectance spectrophotometer 1, "Agronomy Journal, vol. 60, no. 6, pp. 640-643, 1968.

[38] G. Cavallaro, Y. Bazi, F. Melgani, and M. Riedel, "Multi-Scale Convolutional SVM Networks for Multi-Class Classification Problems of Remote Sensing Images," in IGARSS 2019-2019 IEEE International Geoscience and Remote Sensing Symposium, 2019: IEEE, pp. 875-878.

[39] G. Litjens et al., "A survey on deep learning in medical image analysis, "Medical image analysis, vol. 42, pp. 60-88, 2017.

[40] F. Chollet, Keras: The python deep learning library, "Astrophysics source code library, Code record: 1806.022, 2018.

[41] L. Prechelt, "Automatic early stopping using cross validation: quantifying the criteria, "Neural Networks, vol. 11, no. 4, pp. 761-767, 1998.

[42] Tharwat, "Classification assessment methods," Applied Computing and Informatics, 2020. 